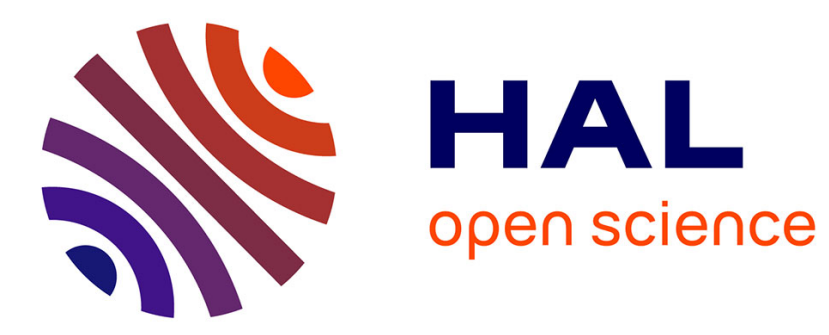

\title{
High-frequency diffraction of a plane electromagnetic wave by an elongated spheroid
}

\author{
Ivan V. Andronov, Daniel Bouche, Marc Duruflé
}

\section{To cite this version:}

Ivan V. Andronov, Daniel Bouche, Marc Duruflé. High-frequency diffraction of a plane electromagnetic wave by an elongated spheroid. IEEE Antennas and Wireless Propagation Letters, 2012, 60 (11), pp.5286-5295. hal-00734640

\section{HAL Id: hal-00734640 \\ https://hal.science/hal-00734640}

Submitted on 11 Oct 2012

HAL is a multi-disciplinary open access archive for the deposit and dissemination of scientific research documents, whether they are published or not. The documents may come from teaching and research institutions in France or abroad, or from public or private research centers.
L'archive ouverte pluridisciplinaire HAL, est destinée au dépôt et à la diffusion de documents scientifiques de niveau recherche, publiés ou non, émanant des établissements d'enseignement et de recherche français ou étrangers, des laboratoires publics ou privés. 


\title{
High-frequency diffraction of a plane electromagnetic wave by an elongated spheroid
}

\author{
Ivan V. Andronov, Daniel P. Bouche and Marc Duruflé
}

\begin{abstract}
An asymptotic formula for the problem of diffraction by a strongly elongated body of revolution is constructed. Its uniform nature with respect to the parameter that characterizes the rate of elongation is demonstrated. The results are in good agreement with numerical simulations.
\end{abstract}

Index Terms-Electromagnetic diffraction, high frequency asymptotics, parabolic wave equation, strongly elongated body.

\section{INTRODUCTION}

$\mathbf{T}$ HE field in the shadow zone of convex bodies illuminated by electromagnetic waves is generated by creeping waves, that propagate along geodesics on the surface of these bodies. The main geometrical parameter governing the propagation constant of these waves is the radius $\rho$ of curvature of the geodesic. However, other geometrical parameters modify the propagation constant of creeping waves. Among these parameters, the curvature of the object transverse to the creeping ray deserves special attention, because, for electromagnetic waves with magnetic field parallel to the surface (called magnetic creeping waves), the transverse curvature decreases attenuation.

More generally, large transverse curvature or sharp edges promote the propagation of waves, as exemplified by the following cases. Senior [1] observed in 1969, while studying diffraction by disks that a wave propagates with low attenuation along the rim of the disk. The Sommerfeld wave [2] propagates with only logarithmic attenuation along conducting wires. Finally, a source on a cylinder excites a wave that propagates along the generatrix of the cylinder, and only decreases as the inverse of the square root of the distance [3].

The impact of transverse curvature on the propagation constant of creeping waves, and more specifically, the case of creeping wave on elongated bodies, has been analysed in [4] (see also [5]). It was shown that, if the transverse radius of curvature is of the same order as the radius of curvature of the geodesic, the propagation constant decreases linearly with transverse curvature. If the transverse curvature is of the order $k^{2 / 3} \rho^{-1 / 3}$, it gives rise to low attenuation modes, whose propagation constant is expressed as a solution of dispersion equations in terms of Heun functions.

I. V. Andronov is with University of Saint Petersburg, Russia, e-mail:iva---@list.ru

D. Bouche is with CEA/DAM Ile de France, Arpajon, France, e-mail:daniel.bouche@cea.fr

M. Duruflé is with Institute of Mathematics, Bordeaux, France
However, for an elongated body, such as a (very) prolate spheroid, illuminated along its axis by an incident wave, the whole object is not in the deep shadow, but in the light shadow transition zone. The extension of the analysis of [4] to this transition zone, aiming to generalize Fock functions for elongated objects was only partly successful. Namely the effect was described correctly at the qualitative level, but agreement with numerical computations was not perfect.

Another approach, in the spirit of the work of Engineer et al [6], who studied diffraction by 2D slender bodies, was initiated in [7]. Here we present a more detailed description of the asymptotic procedure and study the special function which describes the induced current on the surface of elongated body. In particular, we show that, for not too elongated bodies, the formula reduces to Fock asymptotics, while in the opposite limiting case we get the geometrical optics solution.

In section 2 of the paper, using separation of variables, that turns out to be possible for an elongated spheroid, we obtain a solution for the forward going wave. This solution has the form of an integral of Whittaker functions.

In section 3, we use this solution to compute the current on the surface. We check the validity of our approach first by showing that, in the limit of moderately elongated spheroid, our solution reverts to Fock function, and by comparing, in section 4 , the results with numerical computations.

\section{ASYMPTOTIC FORMULA FOR FORWARD GOING WAVE}

We deal with large bodies, such that $k \rho \gg 1$. The assumption that the body is strongly elongated means that

$$
(k \rho)^{2 / 3} \frac{\rho_{t}}{\rho}=O(1),
$$

where $\rho_{t}$ is the radius of transverse curvature. It is worth noting that it follows from (1) that $k \rho_{t}=(k \rho)^{1 / 3}$, that is the radius of transverse curvature is also large compared to the wavelength.

We derive the asymptotics of the field of electromagnetic wave diffraction by a strongly elongated body under the following two assumptions. First, the body has angular symmetry of revolution, and the field is excited by a plane wave axially incident on the body. Second, the surface can be well approximated by spheroidal surface and its deviation from that surface can be accounted for with the next order terms of the asymptotics. However, we derive only the principal order term in this paper. 


\section{A. Coordinate system}

Let us introduce the spheroid, which has the same radii of curvature at the light-shadow boundary as the surface of the elongated body. The semiaxes (small $a$ and large $b$ ) of that spheroid are defined by the formulae

$$
\rho=\frac{b^{2}}{a}, \quad \rho_{t}=a .
$$

Here $\rho$ is the radius of curvature of the body in the longitudinal cross-section on the light-shadow boundary and $\rho_{t}$ is its radius of curvature in transverse cross-section.

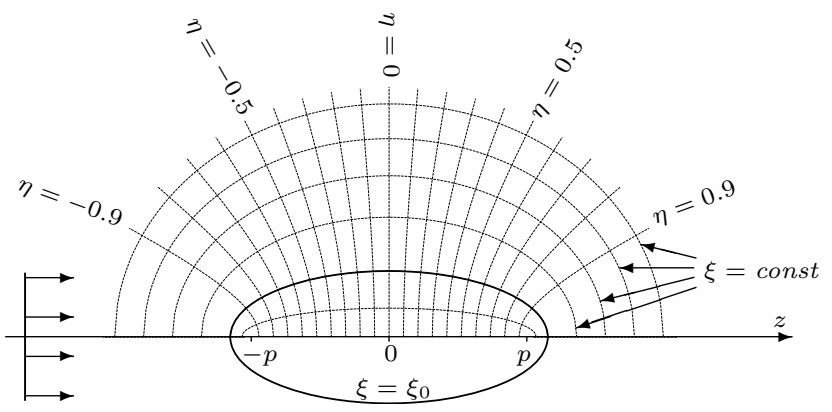

Fig. 1. Geometry of the problem.

We introduce elongated spheroidal coordinates $(\xi, \eta, \varphi)[8]$

$$
z=p \xi \eta, \quad r=p \sqrt{\xi^{2}-1} \sqrt{1-\eta^{2}}, \quad p=\sqrt{b^{2}-a^{2}},
$$

where $(r, \varphi, z)$ are cylindrical coordinates with the $z$ axis coincident with the axis of the spheroid, and $z=0$ in the center of the spheroid (see Fig. 1). Coordinate $\xi$ is called radial and takes values $\xi \geq 1$, coordinate $\eta$ is angular and varies on the interval $-1 \leq \eta \leq 1$. The surface of the spheroid is given by the formula

$$
\xi=\xi_{0} \equiv \frac{b}{\sqrt{b^{2}-a^{2}}} .
$$

Let us introduce the usual asymptotic parameter

$$
m=\left(\frac{k \rho}{2}\right)^{1 / 3}
$$

and the parameter $\kappa$ which characterizes the rate of elongation of the body

$$
\kappa=2 m^{2} \frac{\rho_{t}}{\rho} .
$$

We shall derive an asymptotic expansion for the electromagnetic field in a thin layer near the surface assuming that $m \gg 1$ and $\kappa=O(1)$, which corresponds to the case of a strongly elongated body. Under these two assumptions in the leading order by $m$ we get

$$
\xi_{0}=1+\frac{\kappa}{4 m^{2}} .
$$

\section{B. Maxwell equations}

We introduce vectors $\mathbf{E}=\sqrt{\varepsilon_{0}} \mathcal{E}$ and $\mathbf{H}=\sqrt{\mu_{0}} \mathcal{H}$, where $\mathcal{E}$ and $\mathcal{H}$ are the usual electric and magnetic vectors. Maxwell equations for these vectors

$$
\left\{\begin{array}{l}
\operatorname{rot} \mathbf{E}=i k \mathbf{H} \\
\operatorname{rot} \mathbf{H}=-i k \mathbf{E}
\end{array}\right.
$$

in spheroidal coordinates take the form

$$
\begin{aligned}
\frac{\partial}{\partial \eta}\left(h_{\varphi} E_{\varphi}\right)-\frac{\partial}{\partial \varphi}\left(h_{\eta} E_{\eta}\right) & =-i k h_{\eta} h_{\varphi} H_{\xi}, \\
\frac{\partial}{\partial \varphi}\left(h_{\xi} E_{\xi}\right)-\frac{\partial}{\partial \xi}\left(h_{\varphi} E_{\varphi}\right) & =-i k h_{\varphi} h_{\xi} H_{\eta}, \\
\frac{\partial}{\partial \xi}\left(h_{\eta} E_{\eta}\right)-\frac{\partial}{\partial \eta}\left(h_{\xi} E_{\xi}\right) & =-i k h_{\xi} h_{\eta} H_{\varphi}, \\
\frac{\partial}{\partial \eta}\left(h_{\varphi} H_{\varphi}\right)-\frac{\partial}{\partial \varphi}\left(h_{\eta} H_{\eta}\right) & =i k h_{\eta} h_{\varphi} E_{\xi}, \\
\frac{\partial}{\partial \varphi}\left(h_{\xi} H_{\xi}\right)-\frac{\partial}{\partial \xi}\left(h_{\varphi} H_{\varphi}\right) & =i k h_{\varphi} h_{\xi} E_{\eta}, \\
\frac{\partial}{\partial \xi}\left(h_{\eta} H_{\eta}\right)-\frac{\partial}{\partial \eta}\left(h_{\xi} H_{\xi}\right) & =i k h_{\xi} h_{\eta} E_{\varphi},
\end{aligned}
$$

where $h_{\xi}, h_{\eta}$ and $h_{\varphi}$ are metric coefficients. For the coordinate system of elongated spheroid, the metric coefficients are given by the formulae [8]

$$
\begin{gathered}
h_{\xi}=p \sqrt{\frac{\xi^{2}-\eta^{2}}{\xi^{2}-1}}, \quad h_{\eta}=p \sqrt{\frac{\xi^{2}-\eta^{2}}{1-\eta^{2}}}, \\
h_{\varphi}=p \sqrt{\xi^{2}-1} \sqrt{1-\eta^{2}} .
\end{gathered}
$$

Assuming the field dependence on the angle $\varphi$ in the form of $e^{i \ell \varphi}$ and using equations (9), (10), (12) and (13) we express $E_{\xi}, E_{\eta}, H_{\xi}$ and $H_{\eta}$ via $E_{\varphi}$ and $H_{\varphi}$. Substituting these expressions into equations (11) and (14) yields the system of differential equations for $E_{\varphi}$ and $H_{\varphi}$. We exploit now the symmetry of these two equations, and by setting

$$
E_{\varphi}=\widehat{P}+\widehat{Q}, \quad H \varphi=-i \widehat{P}+i \widehat{Q},
$$

reduce this system to two independent equations. However, these equations are to cumbersome and we de not present them here.

\section{Parabolic equation method}

In order to solve these cumbersome equations we use asymptotic approach. For that we introduce the new radial (normal) coordinate $\nu$ by the formula

$$
\xi=1+\frac{\nu}{4 m^{2}} .
$$

Note that on the surface $\nu=\kappa$.

Further we extract the quick oscillating factor

$$
\hat{P}=\exp \left(i k p \xi_{0} \eta\right) P(\eta, \nu), \quad \hat{Q}=\exp \left(i k p \xi_{0} \eta\right) Q(\eta, \nu)
$$

and sort the terms in the equations for $P$ and $Q$ by the powers of the large parameter $m$. Representing functions $P$ and $Q$ in the form of the series

$$
P=\sum_{j=0} P_{j} m^{-j}, \quad Q=\sum_{j=0} Q_{j} m^{-j}
$$

we get a recurrent system of equations for $P_{j}$ and $Q_{j}$. In the leading order we get

$$
\mathcal{L}_{1-\ell} P_{0}=0, \quad \mathcal{L}_{1+\ell} Q_{0}=0,
$$


where

$$
\begin{aligned}
\mathcal{L}_{n}= & \nu \frac{\partial^{2}}{\partial \nu^{2}}+\frac{\partial}{\partial \nu}+\frac{i}{2} \sqrt{\frac{\kappa}{2}}\left(1-\eta^{2}\right) \frac{\partial}{\partial \eta} \\
& +\frac{1}{8}\left(\kappa \nu-\kappa^{2}\left(1-\eta^{2}\right)-2 i \sqrt{2 \kappa} \eta-\frac{2 n^{2}}{\nu}\right) .
\end{aligned}
$$

Equations (20) are of Schrödinger type, but in Diffraction Theory analogous equations are traditionally called parabolic [10].

\section{Separation of variables}

Equations (20) allow variables separation. Substituting $V(\nu, \eta)=\Phi(\nu) \Psi(\eta)$ into the equation $\mathcal{L}_{n} V=0$ and dividing it by $V$, we get

$$
\begin{aligned}
\nu \frac{\ddot{\Phi}}{\Phi}+\frac{\dot{\Phi}}{\Phi}+ & \frac{\alpha^{2} \nu}{4}-\frac{n^{2}}{4 \nu}= \\
& =-\frac{\alpha}{2}\left(1-\eta^{2}\right) \frac{\dot{\Psi}}{\Psi}+\frac{\alpha^{4}}{2}\left(1-\eta^{2}\right)+\frac{i \alpha}{2} \eta,
\end{aligned}
$$

where dot denotes derivative of a function by its argument and $\alpha=\sqrt{\kappa / 2}$. The left-hand side of (22) depends only on $\nu$ and the right-hand side depends only on $\eta$. Thus they are equal to a constant which we denote as $i \alpha \mu$. The differential equation for function $\Phi(\nu)$ reduces to Whittaker equation [9]

$$
\frac{d^{2} F}{d x^{2}}+\left(-\frac{1}{4}+\frac{\mu}{x}+\frac{1-n^{2}}{4 x^{2}}\right) F=0 .
$$

Denoting its solution as $F_{\mu, n / 2}(x)$ we get

$$
\Phi(\nu)=\frac{1}{\sqrt{\nu}} F_{\mu, n / 2}(-i \alpha \nu) .
$$

The differential equation for the function $\Psi(\eta)$ can be solved in elementary functions

$$
\Psi(\eta)=\frac{\exp \left(-i \alpha^{3} \eta\right)}{\sqrt{1-\eta^{2}}}\left(\frac{1-\eta}{1+\eta}\right)^{\mu} .
$$

A particular solution of equation $\mathcal{L}_{n} U_{n}=0$ can be written as a superposition of elementary solutions $\Phi(\nu) \Psi(\eta)$ in the form of an integral with $\mu$ being the variable of integration

$$
\begin{aligned}
& U_{n}=U_{n}(\Omega, F) \\
= & \frac{\exp \left(-i \alpha^{3} \eta\right)}{\sqrt{\nu} \sqrt{1-\eta^{2}}} \int \Omega(\mu)\left(\frac{1-\eta}{1+\eta}\right)^{\mu} F_{\mu, n / 2}(-i \alpha \nu) d \mu,
\end{aligned}
$$

where $\Omega$ is an arbitrary function. We do not fix the path of integration in (26), it will be chosen later.

Then

$$
P_{0}=U_{1-\ell}, \quad Q_{0}=U_{1+\ell} .
$$

The electromagnetic field has the form of the sum of the incident plane wave and some wave reflected from the surface. This reflected wave is subject to the radiation condition. So, when writing the representation (26) for the reflected wave one needs to choose such a solution of Whittaker's equation which has its asymptotics in the form of an outgoing wave. Such a solution is the Whittaker function $W_{\mu, n / 2}(x)$, having the asymptotics

$$
W_{\mu, n / 2}(x) \sim x^{\mu} e^{-x / 2}, \quad|x| \rightarrow+\infty .
$$

The incident wave can be prolongated inside the spheroid and is finite on the axis, that is at $\nu=0$. So, when using the representation (26) for the incident wave we should choose another solution of Whittaker's equation, the Whittaker function $M_{\mu, n / 2}(x)$, which is regular at zero.

\section{E. Representation of the incident wave}

Consider the plane incident wave

$$
\mathbf{E}^{(i n c)}=E_{0} e^{i k z} \mathbf{e}_{y}, \quad \mathbf{H}^{(i n c)}=-E_{0} e^{i k z} \mathbf{e}_{x},
$$

where $E_{0}$ is the amplitude, $\mathbf{e}_{x}$ are $\mathbf{e}_{y}$ are the unit vectors in Cartesian coordinate system. In the spheroidal coordinate system this wave has the components (same as in cylindrical)

$$
E_{\varphi}^{(i n c)}=E_{0} e^{i k p \xi \eta} \cos \varphi, \quad H_{\varphi}^{(i n c)}=E_{0} e^{i k p \xi \eta} \sin \varphi .
$$

Then for the functions $P, Q$ we get

$$
\begin{aligned}
P_{0}^{(i n c)} & =\frac{E_{0}}{2} e^{i \varphi} \exp \left(\frac{i}{2} \sqrt{\frac{\kappa}{2}}(\nu-\kappa) \eta\right), \\
Q_{0}^{(i n c)} & =\frac{E_{0}}{2} e^{-i \varphi} \exp \left(\frac{i}{2} \sqrt{\frac{\kappa}{2}}(\nu-\kappa) \eta\right) .
\end{aligned}
$$

On the other hand, functions $P^{(i n c)}$ and $Q^{(i n c)}$ can be represented in the form of the solutions (26), where, as noted above, one should set $F_{\mu, n / 2}(-i \alpha \nu)=M_{\mu, n / 2}(-i \alpha \nu)$

$$
P_{0}^{(i n c)}=e^{i \varphi} U_{0}\left(\Omega^{(i n c)}, M\right), \quad Q_{0}^{(i n c)}=e^{-i \varphi} U_{0}\left(\Omega^{(i n c)}, M\right),
$$

where $\Omega(\mu)^{(i n c)}$ is to be determined. To find $\Omega(\mu)^{(i n c)}$ it is sufficient to equate the representations (31) and (32) at a fixed value of coordinate $\nu$. We do that at $\nu=\kappa$, that is on the surface of the spheroid. Then we get the following integral equation

$\frac{\exp \left(-i \alpha^{3} \eta\right)}{\sqrt{\kappa} \sqrt{1-\eta^{2}}} \int \Omega^{(i n c)}(\mu)\left(\frac{1-\eta}{1+\eta}\right)^{\mu} M_{\mu, 0}(-i \alpha \kappa) d \mu=\frac{E_{0}}{2}$.

To simplify this expression, we introduce the new variable $t$ by setting

$$
\eta=\frac{t-1}{t+1} .
$$

When $\eta$ varies from $\eta=-1$ to $\eta=1$, the variable $t$ takes values from zero to infinity. Equation (33) takes the form

$$
\int t^{-\mu} f(\mu) d \mu=\widehat{f}(t),
$$

where

$$
\begin{gathered}
f(\mu)=M_{\mu, 0}(-i \alpha \kappa) \Omega^{(i n c)}(\mu), \\
\widehat{f}(t)=E_{0} \sqrt{\kappa} \frac{\sqrt{t}}{t+1} \exp \left(i \alpha^{3} \frac{t-1}{t+1}\right) .
\end{gathered}
$$

Noting that, if we choose the path of integration along the imaginary axis, the left-hand side of this equation takes the form of inverse Mellin transform [11]. The solution is obtained by direct Mellin transform

$$
f(\mu)=\frac{1}{2 \pi i} \int_{0}^{+\infty} \widehat{f}(t) t^{\mu-1} d t .
$$


Changing the integration variable in this integral to $-\eta$, we note that up to a multiplier this integral coincides with the integral representation of Whittaker function $M_{\mu, 0}(-i \alpha \kappa)$ (see [9]). Thus, we finally get

$$
\Omega^{(i n c)}=E_{0} \frac{e^{-i \pi / 4}}{2 \pi} \frac{1}{\sqrt{\alpha}} \Gamma\left(\frac{1}{2}-\mu\right) \Gamma\left(\frac{1}{2}+\mu\right) .
$$

We shall search the total field of diffraction in the following form with unknown functions $\omega_{0}$ and $\omega_{1}$

$$
\begin{aligned}
& E_{\varphi}(\eta, \nu)=E_{0} \exp (i k p \eta) \frac{e^{-i \pi / 4}}{\pi} \frac{\cos \varphi}{\sqrt{\alpha \nu} \sqrt{1-\eta^{2}}} \times \\
& \times \int_{-i \infty}^{+i \infty} \Gamma\left(\frac{1}{2}-\mu\right) \Gamma\left(\frac{1}{2}+\mu\right)\left(\frac{1-\eta}{1+\eta}\right)^{\mu}\left(M_{\mu, 0}(-i \alpha \nu)\right. \\
& \left.\quad-\omega_{0}(\mu) W_{\mu, 0}(-i \alpha \nu)+\omega_{1}(\mu) W_{\mu, 1}(-i \alpha \nu)\right) d \mu, \quad(40) \\
& H_{\varphi}(\eta, \nu)=E_{0} \exp (i k p \eta) \frac{e^{-i \pi / 4}}{\pi} \frac{\sin \varphi}{\sqrt{\alpha \nu} \sqrt{1-\eta^{2}}} \times \\
& \quad+i \infty \\
& \quad \int_{-i \infty} \Gamma\left(\frac{1}{2}-\mu\right) \Gamma\left(\frac{1}{2}+\mu\right)\left(\frac{1-\eta}{1+\eta}\right)^{\mu}\left(M_{\mu, 0}(-i \alpha \nu)\right. \\
& \left.\quad-\omega_{0}(\mu) W_{\mu, 0}(-i \alpha \nu)-\omega_{1}(\mu) W_{\mu, 1}(-i \alpha \nu)\right) d \mu .
\end{aligned}
$$

The terms containing Whittaker functions $M$ correspond to the incident wave. For the representation of the reflected field we have chosen the same path of integration, so that we can apply the boundary conditions to the subintegral expressions, which will define functions $\omega_{0}$ and $\omega_{1}$.

\section{F. Boundary conditions}

On the surface of the spheroid stretched radial coordinate $\nu$ is equal to the parameter $\kappa$. The boundary conditions on the surface of a perfectly conducting spheroid have the form $E_{\eta}(\eta, \kappa)=0$ and

$$
E_{\varphi}(\eta, \kappa)=0
$$

With the help of the formula expressing $E_{\eta}$ via $E_{\varphi}$ and $H_{\varphi}$ and accounting for (42), the first condition yields

$$
2 \kappa \frac{\partial H_{\varphi}(\eta, \kappa)}{\partial \nu}+H_{\varphi}(\eta, \kappa)=0 .
$$

Substituting representations (40) and (41) into the conditions (42) and (43), we get the system of algebraic equations for the amplitude functions $\omega_{0}$ and $\omega_{1}$

$$
\left\{\begin{array}{l}
\omega_{0} W_{\mu, 0}(-i \alpha \kappa)-\omega_{1} W_{\mu, 1}(-i \alpha \kappa)=M_{\mu, 0}(-i \alpha \kappa), \\
\omega_{0} \dot{W}_{\mu, 0}(-i \alpha \kappa)+\omega_{1} \dot{W}_{\mu, 1}(-i \alpha \kappa)=\dot{M}_{\mu, 0}(-i \alpha \kappa) .
\end{array}\right.
$$

Here and below, dot denotes the derivative of a function with respect to the argument. Solving this system, we find

$$
\begin{aligned}
& \omega_{0}=\frac{M_{\mu, 0}(-i \chi) \dot{W}_{\mu, 1}(-i \chi)+\dot{M}_{\mu, 0}(-i \chi) W_{\mu, 1}(-i \chi)}{W_{\mu, 0}(-i \chi) \dot{W}_{\mu, 1}(-i \chi)+\dot{W}_{\mu, 0}(-i \chi) W_{\mu, 1}(-i \chi)}, \\
& \omega_{1}=\frac{\Gamma^{-1}(1 / 2-\mu)}{W_{\mu, 0}(-i \chi) \dot{W}_{\mu, 1}(-i \chi)+\dot{W}_{\mu, 0}(-i \chi) W_{\mu, 1}(-i \chi)} .
\end{aligned}
$$

In the last formula we introduced $\chi=\alpha \kappa$ and have taken into account the expression for the Wronskian of Whittaker functions, which can be easily derived by using the formulae 13.1.22 and 13.1.32 from [9],

$$
\dot{M}_{\mu, 0} W_{\mu, 0}-M_{\mu, 0} \dot{W}_{\mu, 0}=\Gamma^{-1}(1 / 2-\mu) \text {. }
$$

\section{THE ELECTRIC CURRENT ON THE SURFACE}

\section{A. Special function}

The induced current $\mathbf{J}$ is given by the vector product of the unit vector of the normal to the surface and the magnetic field $\mathbf{H}$ on the surface. The longitudinal component of magnetic vector is asymptotically small (electromagnetic field is transversal in the leading order). Therefore the main component of the current is its longitudinal component which we denote as $J$. It coincides with $H_{\varphi}$ on the surface. Substituting expressions (45) and (46) into representation (41) and setting $\nu=\kappa$, we can find the current on the surface. We represent it by introducing a special function

$$
\begin{aligned}
& A(\eta ; \chi)=\frac{2 e^{-i \pi / 4}}{\pi} \frac{e^{-i \chi \eta / 2}}{\sqrt{\chi} \sqrt{1-\eta^{2}}} \int_{-i \infty}^{+i \infty}\left(\frac{1-\eta}{1+\eta}\right)^{\mu} \times \\
& \times \frac{\Gamma(1 / 2+\mu) W_{\mu, 1}(-i \chi)}{W_{\mu, 0}(-i \chi) \dot{W}_{\mu, 1}(-i \chi)+\dot{W}_{\mu, 0}(-i \chi) W_{\mu, 1}(-i \chi)} d \mu
\end{aligned}
$$

of two variables $\eta$ and $\chi$. The current of the forward going wave is given in the leading order approximation by $m \gg 1$ by the formula

$$
J=E_{0} e^{i k z} \sin (\varphi) A\left(\frac{z}{\sqrt{\rho \rho_{t}}} ; k \rho_{t} \sqrt{\frac{\rho_{t}}{\rho}}\right) .
$$

Here we expressed

$$
\eta=\frac{z}{b}, \quad \text { and } \quad \chi=\frac{k a^{2}}{b}
$$

via the initial parameters of the problem.

To compute the Whittaker function involved in the definition (48) of the special function $A$, we can use the program developed in [12]. For that, we introduce new variable of integration $t=i \mu$ and use the relation [12]

$$
\begin{aligned}
& W_{-i t, \lambda+\frac{1}{2}}(-i \chi)= \exp \left(i \frac{\pi}{2} \lambda-\frac{\pi}{2} t\right) \times \\
& \times \sqrt{\frac{\Gamma(1+\lambda-i t)}{\Gamma(1+\lambda+i t)}} H_{\lambda}^{+}\left(t, \frac{\chi}{2}\right)
\end{aligned}
$$

which expresses Whittaker function via Coulomb wave function $H_{\lambda}^{+}$. After applying the symmetry formula for Gamma function we get

$$
A(\eta, \chi)=\frac{4}{\sqrt{\pi}} \frac{e^{-i \chi \eta / 2}}{\sqrt{\chi} \sqrt{1-\eta^{2}}} \int_{-\infty}^{+\infty}\left(\frac{1+\eta}{1-\eta}\right)^{i t} \Omega(t) d t
$$


where

$$
\begin{aligned}
& \Omega(t)=\frac{e^{\pi t / 2}}{\sqrt{\cosh (\pi t)}} \times \\
& \times\left(\dot{H}_{-1 / 2}^{+}(t, \chi / 2)+\frac{\dot{H}_{1 / 2}^{+}(t, \chi / 2)}{H_{1 / 2}^{+}(t, \chi / 2)} H_{-1 / 2}^{+}(t, \chi / 2)\right)^{-1} .
\end{aligned}
$$

\section{B. Asymptotics of special function A for large $\chi$}

When $\chi$ increases, formula (49) should turn into the usual Fock asymptotics

$$
J=e^{i k s} g(\sigma),
$$

where $s$ is the arc-length measured from the light-shadow boundary, $g(\sigma)$ is the Fock function [13]

$$
g(\sigma)=\frac{1}{\sqrt{\pi}} \int e^{i \sigma \zeta} \frac{1}{\dot{w}_{1}(\zeta)} d \zeta, \quad \text { and } \quad \sigma=m \frac{s}{\rho} .
$$

Here $\dot{w}_{1}(\zeta)$ is the derivative of the Airy function $w_{1}(\zeta)$ in V.A.Fock notations (see formula (62) below).

To prove this, we first express $z$ in terms of the arc-length $s$ by the well-known formula valid in Fock's domain where the radius $\rho$ can be approximated by its constant value at lightshadow boundary. We have

$$
z=s-\frac{s^{3}}{6 \rho^{2}}+\ldots
$$

This allows us to express the first argument of the special function $A$ in the two leading orders by $\chi$ as

$$
\eta \approx\left(\frac{2}{\chi}\right)^{1 / 3} \sigma-\frac{\sigma^{3}}{3 k \sqrt{\rho \rho_{t}}} .
$$

Then, for small $\eta$ we use the decomposition

$\log \left(\frac{1+\eta}{1-\eta}\right) \sim 2 \eta+\frac{2}{3} \eta^{3} \sim 2 \sigma\left(\frac{2}{\chi}\right)^{1 / 3}+\frac{4}{3} \frac{\sigma^{3}}{\chi}-\frac{2}{3} \frac{\sigma^{3}}{k \sqrt{\rho \rho_{t}}}$.

Now, we consider the asymptotics of the Coulomb wave functions $H_{\ell}^{+}(t, \chi / 2)$ for $t \gg 1,2 t \sim \chi / 2$ (see [9], formula 14.6.12). We have

$$
\begin{aligned}
& H_{\ell}^{+}(t, \chi / 2)=G_{\ell}(t, \chi / 2)+i F_{\ell}(t, \chi / 2) \\
& \quad \sim \sqrt{\pi}\left\{\frac{t_{\ell}}{1+\ell(\ell+1) t_{\ell}^{-2}}\right\}^{1 / 6}(\operatorname{Bi}(\xi)+i \operatorname{Ai}(\xi)),
\end{aligned}
$$

where $\mathrm{Ai}$ and $\mathrm{Bi}$ are Airy functions and

$t_{\ell}=t+\sqrt{t^{2}+\ell(\ell+1)}, \quad \xi=\left(t_{\ell}-t\right)\left(\frac{1}{t_{\ell}}+\frac{\ell(\ell+1)}{t_{\ell}^{3}}\right)^{1 / 3}$.

For small values of $\ell$, formulae (60) simplify to

$$
t_{\ell} \approx 2 t, \quad \xi \approx\left(2 t-\frac{\chi}{2}\right)\left(\frac{2}{\chi}\right)^{1 / 3} .
$$

We also use relations for Airy functions, in particular

$$
\sqrt{\pi} \operatorname{Ai}(x)=v(x), \quad v\left(x e^{2 \pi i / 3}\right)=\frac{1}{2} e^{-\pi i / 6} w_{1}(x),
$$

and get

$$
H_{\ell}^{+}(t, \chi / 2) \sim-(2 t)^{1 / 6} w_{1}(\xi) .
$$

The asymptotics (63) allows us to find the asymptotics of the density in the integral representation of the special function $A$. We find

$$
\Omega(t) \sim \frac{1}{\sqrt{2}}\left(\frac{\chi}{2}\right)^{1 / 6} \frac{1}{\dot{w}_{1}(\xi)} .
$$

Finally we change the variable of integration from $t$ to $\xi$ and, after simple derivations, we obtain the desired asymptotics

$$
A(\eta, \chi) \sim \frac{1}{\sqrt{\pi}} e^{i \sigma^{3} / 3} \int e^{i \xi \sigma} \frac{d \xi}{\dot{w}_{1}(\xi)},
$$

that is

$$
A(\eta, \chi) \sim \exp \left(i \frac{\chi \eta^{3}}{6}\right) g(\sigma), \quad \chi \rightarrow+\infty .
$$

This shows that the asymptotic formula (49) indeed transforms to the usual Fock asymptotics.

\section{Asymptotics for the special function $A(\eta, \chi)$ for small $\chi$}

In this section we derive the asymptotics for the special function $A(\eta, \chi)$ for small values of $\chi$. The expression (48) for the function $A(\eta, \chi)$ in terms of Whittaker functions appears more suitable for that purpose. We shall need a representation of Whittaker functions of small argument. To find it, we use formulae 13.1.33 and 13.1.6 of [9] and get

$$
\begin{gathered}
W_{\mu, 0}(z)=-\frac{z^{1 / 2}}{\Gamma(1 / 2-\mu)}\left[\log (z)+\Psi\left(\frac{1}{2}-\mu\right)+2 \gamma\right]+\ldots, \\
W_{\mu, 1}(z)=\frac{-z^{3 / 2}}{2 \Gamma(-1 / 2-\mu)}\left[\log (z)+\Psi\left(\frac{3}{2}-\mu\right)\right. \\
\left.\quad+2 \gamma-\frac{3}{2}\right]+\frac{1}{\Gamma(3 / 2-\mu)} z^{-1 / 2}+\ldots \quad(68)
\end{gathered}
$$

Here $\Psi$ is psi-function, $\gamma$ is the Euler constant and dots denote terms wanishing at $z=0$. Substituting these approximations into (48), we get in the leading order by $\chi \rightarrow 0$ the following expression

$$
\begin{array}{r}
A(\eta, \chi) \sim \frac{2 i}{\pi \sqrt{1-\eta^{2}}} \int_{-i \infty}^{+i \infty} \Gamma\left(\frac{1}{2}+\mu\right) \Gamma\left(\frac{1}{2}-\mu\right) \times \\
\times\left(\frac{1-\eta}{1+\eta}\right)^{\mu} d \mu .
\end{array}
$$

Now we use the symmetry property of Gamma function and change the variable of integration to $t=-i \mu$. This gives

$$
A(\eta, \chi)=\frac{-2}{\sqrt{1-\eta^{2}}} \int_{-\infty}^{+\infty}\left(\frac{1-\eta}{1+\eta}\right)^{i t} \frac{d t}{\cosh (\pi t)} .
$$

Finally, we change the integration variable to $\tau=\pi t$ and rewrite the integral as the integral along the positive semiaxis. This results in the following asymptotics of the special function $A(\eta, \chi)$ for small $\chi$ :

$$
A(\eta, \chi) \sim-\frac{4}{\pi} \frac{1}{\sqrt{1-\eta^{2}}} \int_{0}^{+\infty} \frac{\cos (\theta \tau)}{\cosh (\tau)} d \tau,
$$


where

$$
\theta=\frac{1}{\pi} \log \left(\frac{1-\eta}{1+\eta}\right) .
$$

The integral in (71) can be found explicitly

$$
\int_{0}^{+\infty} \frac{\cos (\theta \tau)}{\cosh (\tau)} d \tau=\frac{\pi}{2} \operatorname{sech}\left(\frac{\pi \theta}{2}\right)
$$

which, due to $\eta=-\tanh (\pi \theta / 2)$, allows to rewrite the asymptotics (71) as

$$
A(\eta, \chi) \underset{\chi \rightarrow 0}{\sim}-2
$$

That means that we get the current of geometrical optics, which is the physically expected result for extremely elongated body. In this case, the whole surface of the body is very close to the illuminated domain, and geometrical optics is the expected limit. This result may also have some relation to Sommerfeld wave, in the limit of perfectly conducting cylinder.

\section{NUMERICAL RESULTS}

\section{A. Conversion of the integrals}

Numerical analysis shows that function $\Omega(t)$ rapidly decreases when $t \rightarrow \pm \infty$. Figure 2 presents the absolute values of $\Omega(t)$ for different values of the parameter $\chi$. It can be seen from Fig. 2, that only a finite interval of $t$ gives the main contribution to the integral. The lower boundary of this interval does not depend on $\chi$ and can be taken equal to -5 to achieve sufficiently high accuracy. The upper bound increases with $\chi$ and we take it equal to $\chi / 2+5$.

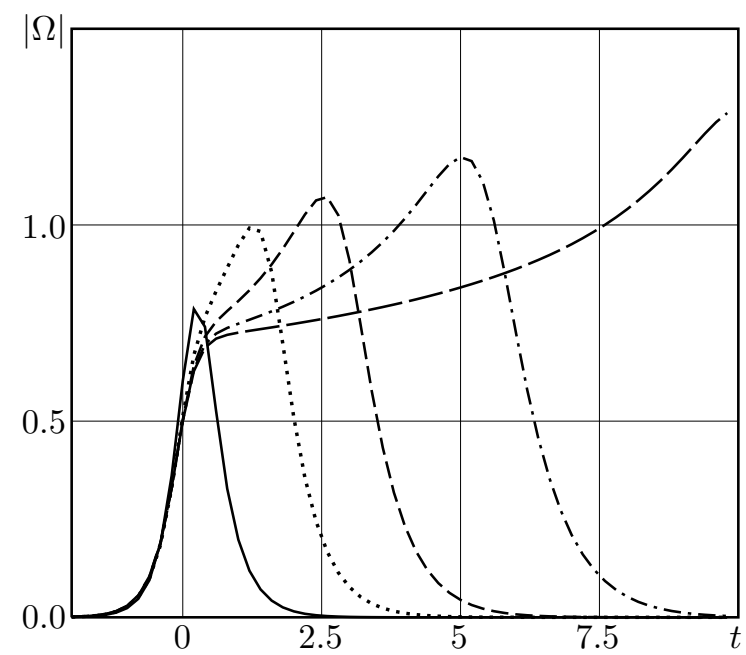

Fig. 2. Absolute values of $|\Omega(t)|$ for $\chi=1$ (solid line), 5 (doted), 10 (dashed), 20 (dash-doted) and 40 (long dashes).

\section{B. Reference solutions}

Reference solutions have been computed by considering Maxwell's equations in axisymmetric domains. The computational domain is made of quadrilaterals, and a finite element method is used to compute the electric field $E$, and magnetic field $H$. These fields can be decomposed in their Fourier series:

$$
E=\sum_{m=-\infty}^{\infty}\left(\begin{array}{c}
E_{r} \\
E_{\varphi} \\
E_{z}
\end{array}\right) e^{-i m \varphi}, \quad H=\sum_{m=-\infty}^{\infty}\left(\begin{array}{c}
H_{r} \\
H_{\varphi} \\
H_{z}
\end{array}\right) e^{-i m \varphi}
$$

Maxwell's equations then have the form

$$
\begin{aligned}
& \left\{\begin{array}{l}
-i \omega \varepsilon r E_{r}+i m H_{z}+\frac{\partial\left(r H_{\varphi}\right)}{\partial z}=0 \\
-i \omega \varepsilon r E_{\varphi}+r\left(\frac{\partial H_{z}}{\partial r}-\frac{\partial H_{r}}{\partial z}\right)=0 \\
-i \omega \varepsilon r E_{z}-i m H_{r}-\frac{\partial\left(r H_{\varphi}\right)}{\partial r}=0
\end{array}\right. \\
& \left\{\begin{array}{l}
-i \omega \mu r H_{r}-i m E_{z}-\frac{\partial\left(r E_{\varphi}\right)}{\partial z}=0 \\
-i \omega \mu r H_{\varphi}-r\left(\frac{\partial E_{z}}{\partial r}-\frac{\partial E_{r}}{\partial z}\right)=0 \\
-i \omega \mu r H_{z}+i m E_{r}+\frac{\partial\left(r E_{\varphi}\right)}{\partial r}=0
\end{array}\right.
\end{aligned}
$$

We introduce the following notations

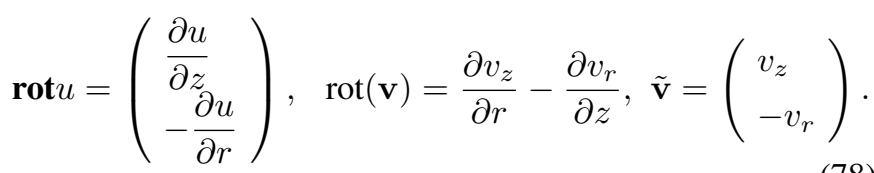

By denoting the new unknowns :

$$
\mathbf{E}=\left(\begin{array}{c}
E_{r} \\
E_{z}
\end{array}\right), \mathbf{H}=\left(\begin{array}{c}
H_{r} \\
H_{z}
\end{array}\right), \tilde{E}_{\varphi}=i E_{\varphi}, \tilde{H}_{\varphi}=i H_{\varphi},
$$

the following system of equations is obtained

$$
\left\{\begin{array}{lll}
-\omega^{2} \varepsilon r \mathbf{E}+m \tilde{\mathbf{H}}-\operatorname{rot}\left(r \tilde{H}_{\varphi}\right) & =0 \\
-\omega^{2} \varepsilon r \tilde{E}_{\varphi}+r \operatorname{rot}(\mathbf{H}) & =0 \\
\mu r \mathbf{H}+m \tilde{\mathbf{E}}-\operatorname{rot}\left(r \tilde{E}_{\varphi}\right) & =0 \\
\mu r \tilde{H}_{\varphi}+r \operatorname{rot}(\mathbf{E}) & =0
\end{array}\right.
$$

The advantage of such a system is that the imaginary part in the variational formulation appears only from either absorbing media, absorbing boundary condition or PML layers. The variational formulation without boundary terms reads : for any functions $\theta, \psi, \phi$ and $\lambda$

$$
\begin{cases}\int_{\Omega}\left(-\omega^{2} \varepsilon r \mathbf{E} \cdot \theta+m \theta \times \mathbf{H}-r \tilde{H}_{\varphi} \operatorname{rot}(\theta)\right) d x & =0 \\ \int_{\Omega}\left(-\omega^{2} \varepsilon r \tilde{E}_{\varphi} \cdot \psi+\mathbf{H} \cdot \operatorname{rot}(r \psi)\right) d x & =0 \\ \int_{\Omega}\left(-\mu r \mathbf{H} \cdot \phi+m \mathbf{E} \times \phi+\phi \cdot \operatorname{rot}\left(r \theta E_{\varphi}\right)\right) d x & =0 \\ \int_{\Omega}\left(-\mu r \tilde{H}_{\varphi} \lambda-r \lambda \operatorname{rot}(\mathbf{E})\right) d x & =0\end{cases}
$$


This variational formulation is symmetric. We consider the following polynomial space :

$$
\mathbb{Q}_{m, n}=\left\{\sum_{i=0}^{m} \sum_{j=0}^{n} a_{i, j} \hat{x}^{i} \hat{y}^{j}, \quad a_{i, j} \in \mathbb{C}\right\}
$$

If we denote by $F$ the mapping from unit square $\hat{K}=[0,1]^{2}$ to a quadrilateral $K$, the associated finite element spaces are equal to :

$$
\begin{gathered}
\mathbf{E} \in V_{E}=\left\{E \in H(\operatorname{curl}, \Omega),\left.D F^{*} E \circ F\right|_{K} \in \mathbb{Q}_{r-1, r} \times \mathbb{Q}_{r, r-1}\right\}, \\
\tilde{E}_{\varphi} \in V_{E_{\varphi}}=\left\{u \in H^{1}(\Omega),\left.u \circ F\right|_{K} \in Q_{r, r}\right\}, \\
\mathbf{H} \in V_{H}=\left\{H \in L^{2}(\Omega),\left.D F^{*} H \circ F\right|_{K} \in \mathbb{Q}_{r, r} \times \mathbb{Q}_{r, r}\right\}, \\
\tilde{H}_{\varphi} \in V_{H_{\varphi}}=\left\{u \in L^{2}(\Omega),\left.u \circ F\right|_{K} \in Q_{r-1, r-1}\right\} .
\end{gathered}
$$

We remark that unknown $\mathbf{E}$ is discretized with elements of Nedelec's first family [14] whereas $\tilde{E}_{\varphi}$ is discretized with usual continuous finite elements [15]. The unknowns $\mathbf{H}$ and $\tilde{H}_{\varphi}$ are local, therefore eliminated through static condensation (by computing the Schur complement associated with unknowns $\mathbf{E}$ and $\tilde{E}_{\varphi}$ ). The resulting linear system is solved by a direct solver. The mesh (see Fig. 3) consists only of quadrilaterals, with curved quadrilaterals in order to fit the geometry. A local refinement is performed near the tip of

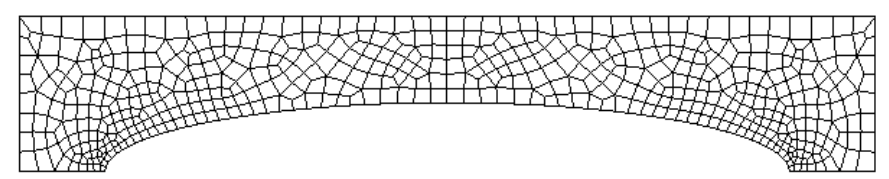

Fig. 3. Typical mesh used for the experiments.

the ellipse, when $a$ is small. The computational domain is surrounded by PML layers (not displayed in the graph). The typical order of approximation $r$ is equal to $r=8$ so that the reference solution is quite accurate. Since the incident plane wave is axial (the wave vector is oriented in $z$-axis), only two modes are involved $(m=-1$, and $m=+1)$.

\section{Comparison with test results}

We consider four spheroids at two frequencies. The parameters are presented in Table I. The problems for spheroid no. 1 at frequency of $1 \mathrm{GHz}$ is equivalent to the problem for spheroid no. 2 at frequency of $2 \mathrm{GHz}$.

TABLE I

TEST PROBLEMS PARAMETERS.

\begin{tabular}{|c|c|l|l|r|r|}
\hline no. & $f(\mathrm{GHz})$ & $a(\mathrm{~m})$ & \multicolumn{1}{c|}{$b(\mathrm{~m})$} & \multicolumn{1}{c|}{$\chi$} & \multicolumn{1}{c|}{$k b$} \\
\hline 1 & 1 & 1.0 & 2.5 & 8.38338 & 52.39613 \\
1 & 2 & 1.0 & 2.5 & 16.76676 & 104.79225 \\
2 & 1 & 0.5 & 1.25 & 4.19169 & 26.19806 \\
2 & 2 & 0.5 & 1.25 & 8.38338 & 52.39613 \\
3 & 1 & 0.5 & 1.76776695 & 2.96397 & 37.04966 \\
3 & 2 & 0.5 & 1.76776695 & 5.92795 & 74.09931 \\
4 & 1 & 0.3125 & 1.39754249 & 1.46452 & 29.29032 \\
4 & 2 & 0.3125 & 1.39754249 & 2.92903 & 58.58065 \\
\hline
\end{tabular}
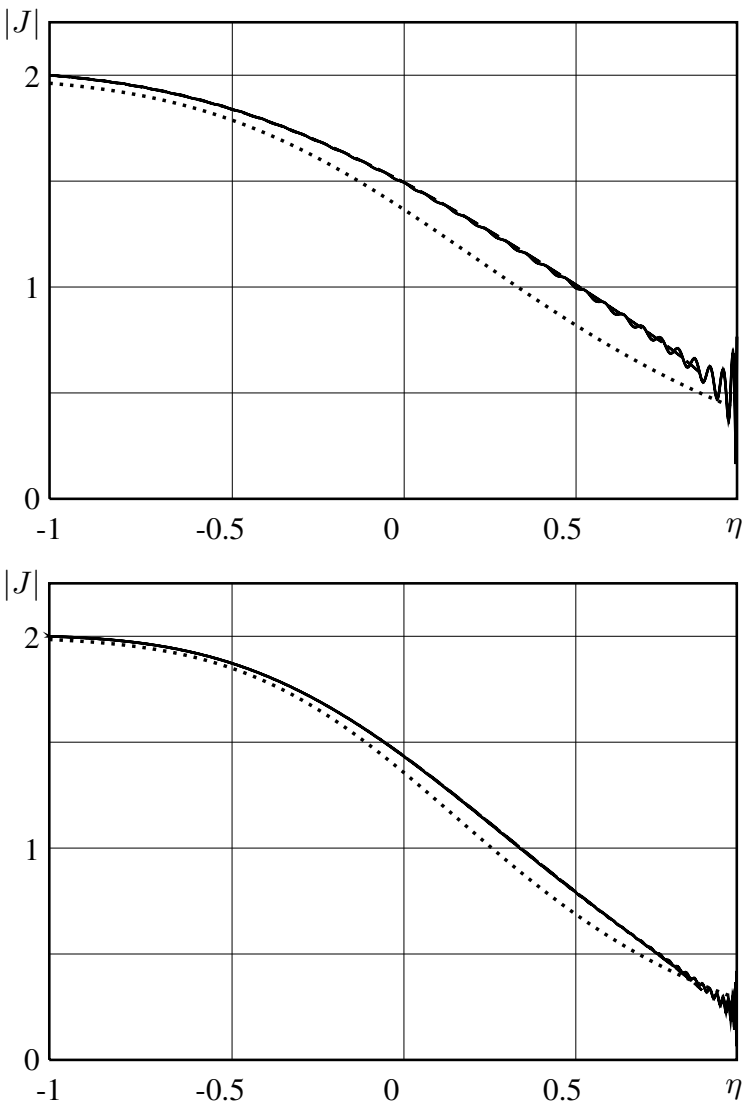

Fig. 4. Absolute values of test current and current of forward going wave at frequencies of $1 \mathrm{GHz}$ (upper) and $2 \mathrm{GHz}$ (lower) on spheroid no. 1.

Absolute values of the current are presented on Figs. 4-7. We accept the amplitude of the incident plane wave $E_{0}=1$. Numerical results are given by solid curves, results computed by asymptotic formula are given by dashed curves. The doted curves present Fock asymptotics (54) which does not take into account the elongation of the body. We see good coincidence of our asymptotic formula with numerical results, while the standard Fock asymptotics gives much underestimated approximation. The curves computed with the use of the asymptotics (52) give a kind of mean value of oscillating curves computed numerically. These oscillations are due to the interference of forward and backward going wave and can be reproduced when taking into account not only the forward going wave, but backward going wave as well.

We conclude that the asymptotic formula (52) describes the absolute values of the currents on the surface of the perfectly conducting spheroids pretty well. To compare the phases, we present on Figs. 8-11 phase differences $\delta \Psi=$ $\arg (J)-\arg \left(J^{\text {num }}\right)$ between the values of the current found asymptotically $(J)$ and numerically $\left(J^{\text {num }}\right)$. These figures also show good agreement between asymptotic and numerical results.

The difference of asymptotic and numerical results, in fact, is the result of two factors. First, we used only the principal order term of the asymptotic formula. The correction due to next order terms should decrease with frequency. This can be seen from the comparison of the errors for the frequency of $1 \mathrm{GHz}$ (on the left-hand side graphics) and for the frequency 

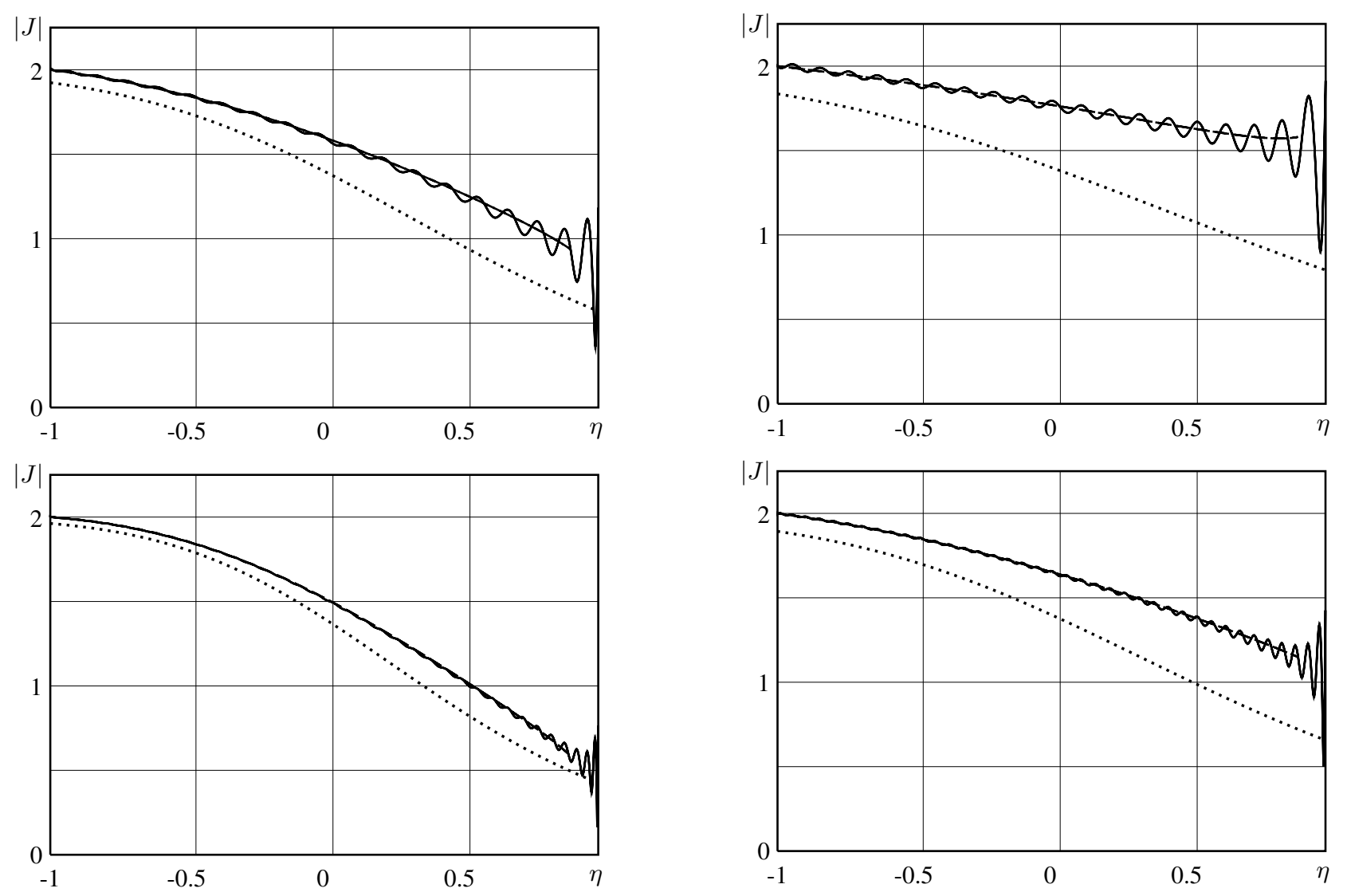

Fig. 5. Absolute values of test current and current of forward going wave at frequencies of $1 \mathrm{GHz}$ (upper) and $2 \mathrm{GHz}$ (lower) on spheroid no. 2.
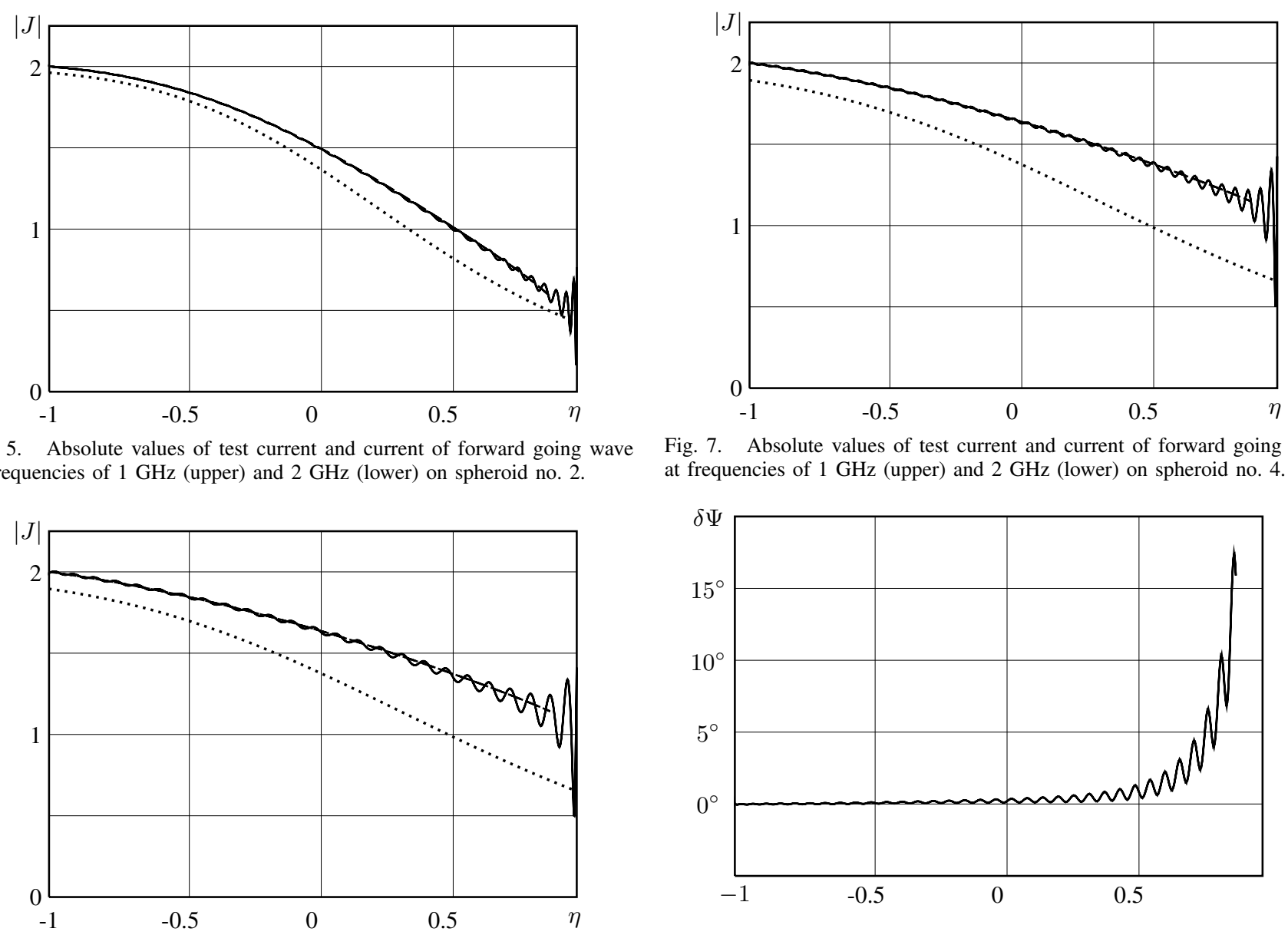

Fig. 7. Absolute values of test current and current of forward going wave at frequencies of $1 \mathrm{GHz}$ (upper) and $2 \mathrm{GHz}$ (lower) on spheroid no. 4 .
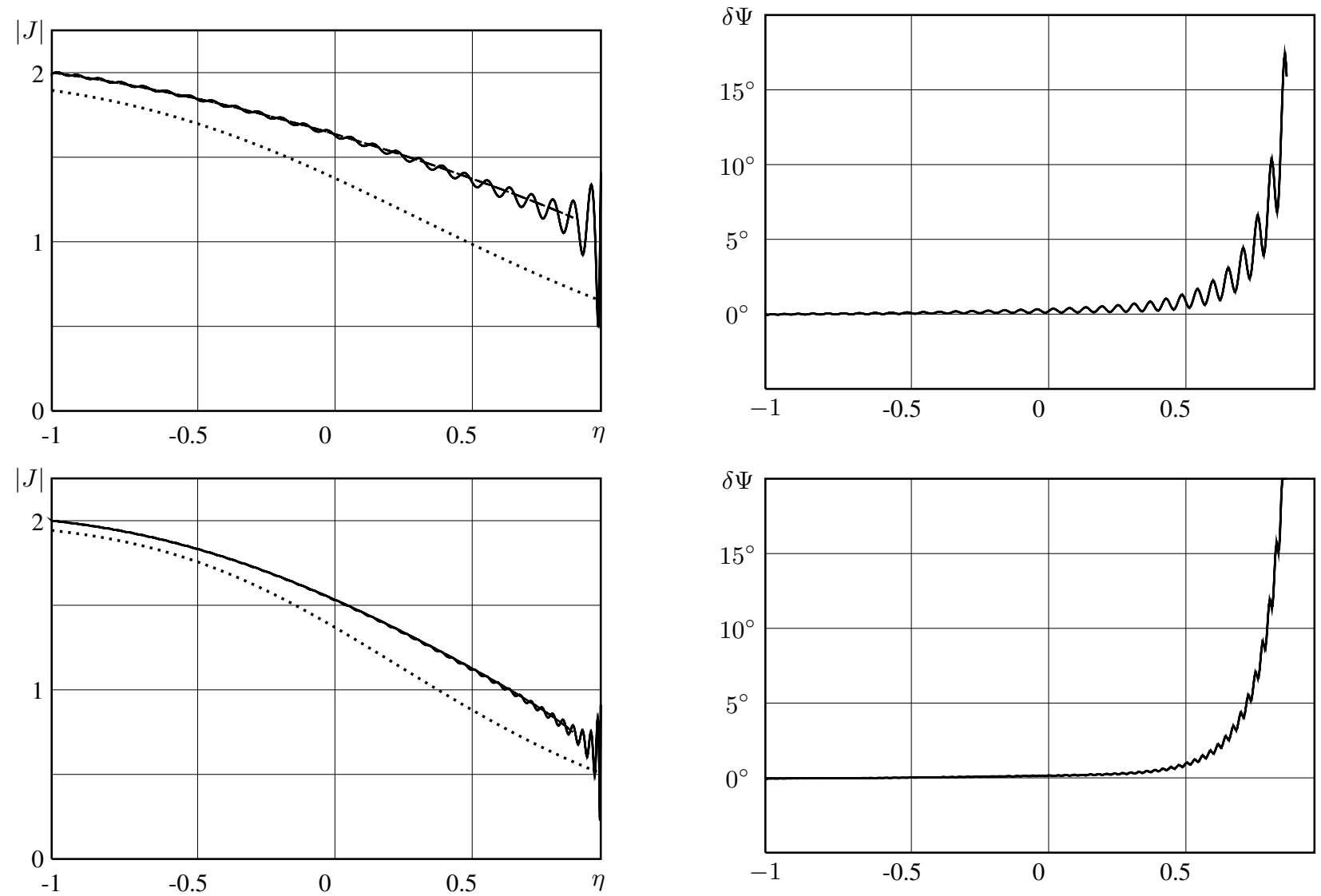

Fig. 6. Absolute values of test current and current of forward going wave at frequencies of $1 \mathrm{GHz}$ (upper) and $2 \mathrm{GHz}$ (lower) on spheroid no. 3 .

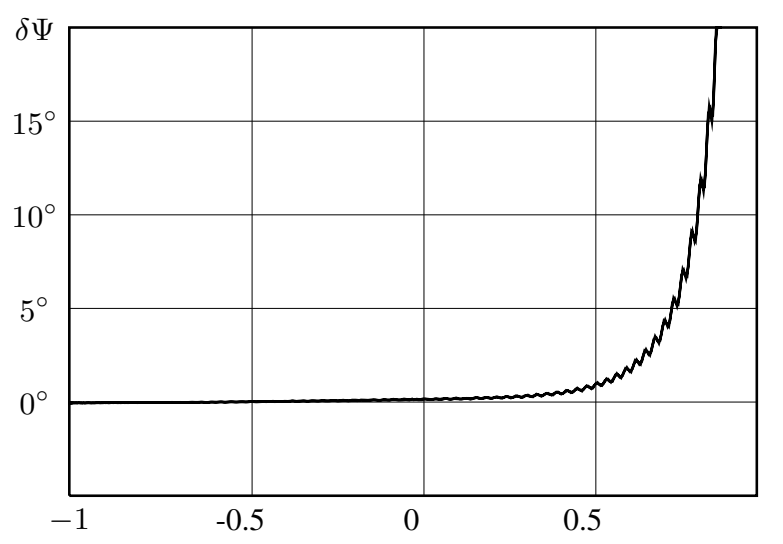

Fig. 8. Phase errors for spheroid no. 1 at frequencies of $1 \mathrm{GHz}$ (upper) and $2 \mathrm{GHz}$ (lower). 

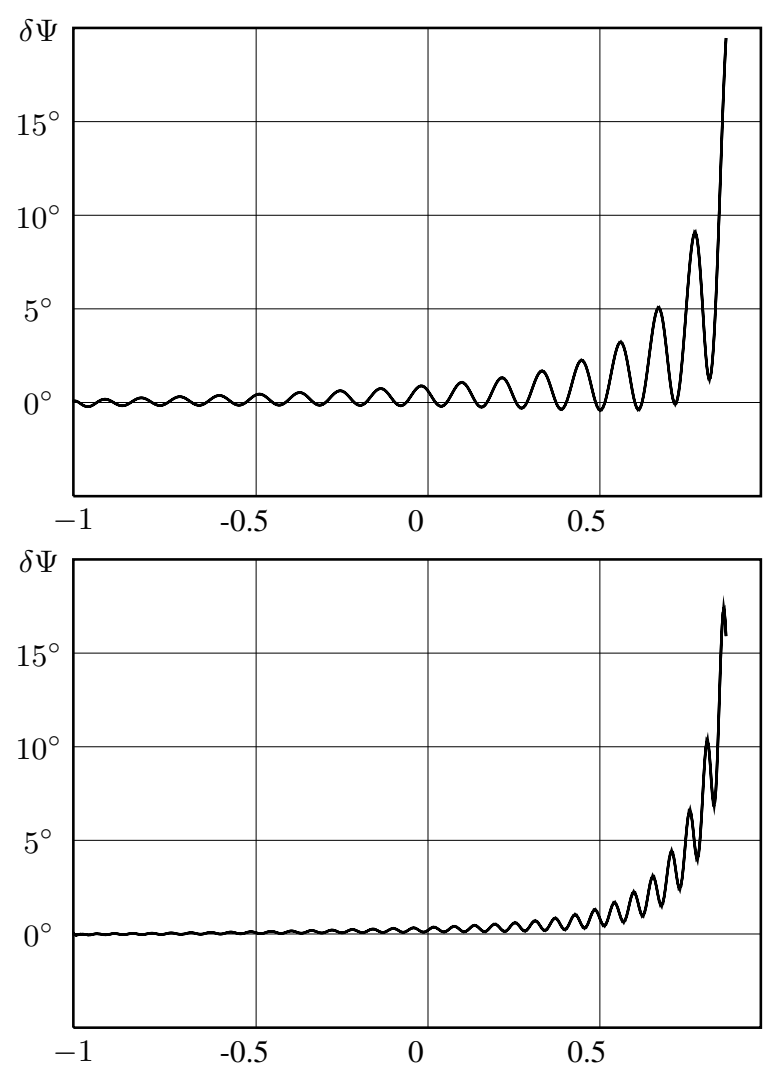

Fig. 9. Phase errors for spheroid no. 2 at frequencies of $1 \mathrm{GHz}$ (upper) and $2 \mathrm{GHz}$ (lower).
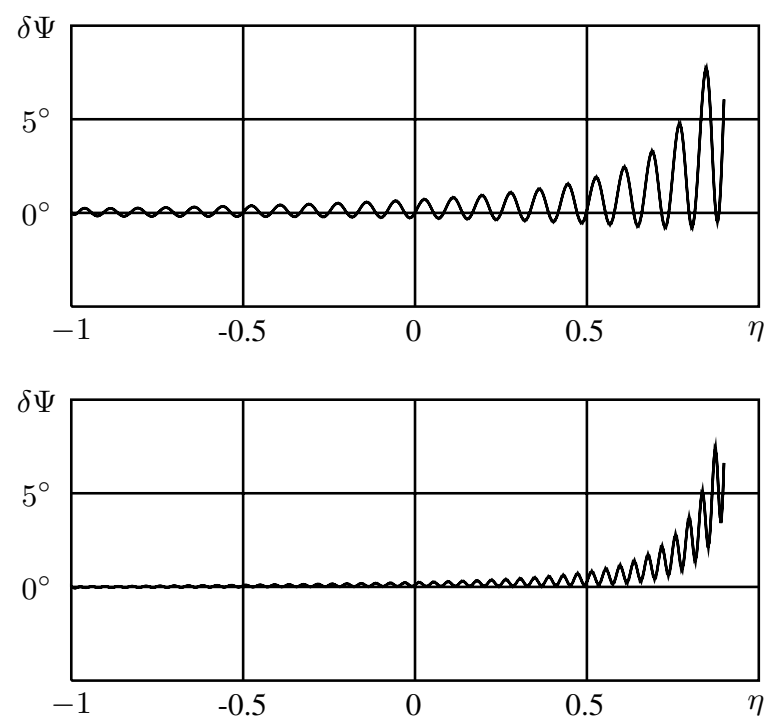

Fig. 10. Phase errors for spheroid no. 3 at frequencies of $1 \mathrm{GHz}$ (upper) and $2 \mathrm{GHz}$ (lower).

of $2 \mathrm{GHz}$ (right-hand side graphics). The second part is due to the fact that the total electromagnetic field is the sum of two wave processes. The first of them propagates in forward direction, the other is formed by reflection of the forward wave by the shadowed extremity of the spheroid and propagates in the backward direction. The attenuation of waves decreases with the increase the parameter $\chi$ and, as a result, the backward going wave contributes more on the more elongated spheroids.
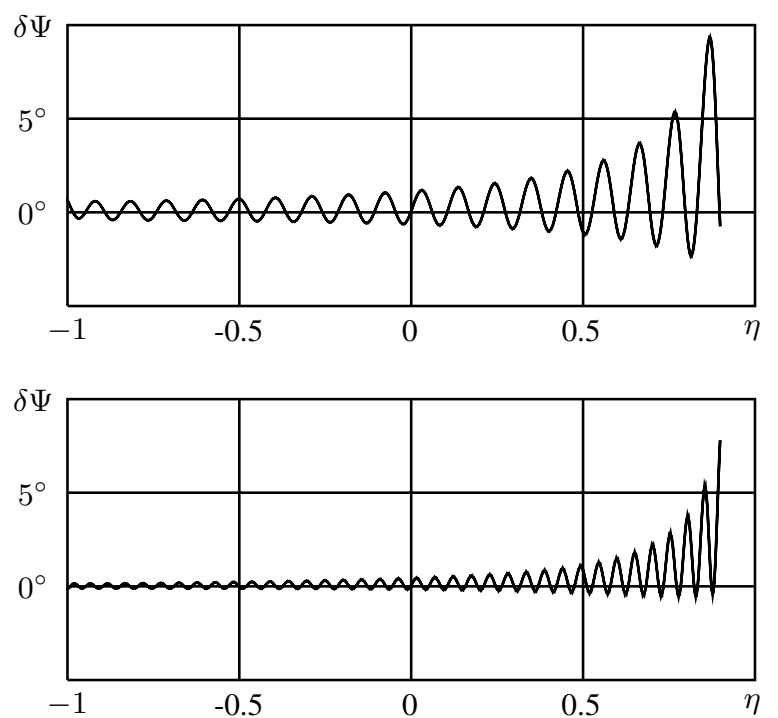

Fig. 11. Phase errors for spheroid no. 4 at frequencies of $1 \mathrm{GHz}$ (upper) and $2 \mathrm{GHz}$ (lower).

We see that by comparing results for spheroids from no. 1 to no. 4.

We see also that the error increases at the shadowed extremity of spheroids. This is natural, because when deriving the asymptotic formula, we assumed that the quick oscillating factor is $e^{i k z}$, which is true only in the middle domain of spheroids. Further, we have not taken into account the effect of focusing which takes place at the shadowed extremity of spheroids.

Some error especially in the phase can be also present in the numerical results, with which we compare our asymptotic approximation.

\section{CONClusion}

We have derived an asymptotic formula for the currents on an elongated spheroid, which shows good coincidence with numerical results. It improves the usual Fock asymptotics by taking into account the effect of transverse curvature and allows to consider diffraction on both not so elongated and very elongated bodies. In the limit of not elongated body, this new asymptotics reduces to Fock results. In the opposite limit of extremely elongated body, we get a wave which does not attenuate, and obtain the current of geometrical optics.

The domain of applicability of the new asymptotic formula is restricted to a region in the middle part of the surface. When the observation point approaches to the shadowed extremity, the asymptotic formula gives diverging results. Though we have not derived the next order term of the asymptotic expansion which is $1 / m$ times smaller, we expect that the order of the singularity at $\eta=1$ will increase at each next order and due to that the derived approximation looses its asymptotic character when $1-\eta=O(1 / m)$.

Analysis of the lower bound of the frequency domain, where the derived approximation is applicable, requires additional comparison with numerical results. Note that the minimal value of asymptotic parameter $m$ for the presented in this 
paper tests is $\approx 3.2$, but we expect that the formula can be used for smaller values of $m$, too.

\section{REFERENCES}

[1] T. B. A. Senior, Disk scattering at edge-on incidence, IEEE Trans Antennas Propag, 17(6) (1969) 751-756.

[2] T. B. A. Senior, Loop excitation of travelling waves, Can J Phys, 40 (1962) 1736.

[3] T. S. Bird, Comparison of Asymptotic Solutions for the Surface Field Excited by a Magnetic Dipole on a Cylinder, IEEE Transactions on Antennas and Propagation, AP-32(11) (1984) 1237-1244.

[4] I. V. Andronov, D. Bouche, Asymptotic of creeping waves on a strongly prolate body, Ann. Télécommun. 49(3-4) (1994) 205-210.

[5] I. V. Andronov, D. Bouche, F. Molinet, Asymptotic and hybrid methods in electromagnetics, IEE, London, 2005.

[6] J. C. Engineer, J. R. King, R. H. Tew, Diffraction by slender bodies, Eur J Appl Math, 9(2) (1998) 129-158.

[7] I. V. Andronov "High-frequency Asymptotics for Diffraction by a Strongly Elongated Body", IEEE Antennas Wirel Propag Lett 8 (2009) 872.

[8] I. V. Komarov, L. I. Ponomarev, S. Yu. Slavyanov, Spheroidal and Coulomb spheroidal functions, Moscow, Science, 1976 (in Russian).

[9] Handbook of mathematical functions, Ed. M.Abramowitz, I.Stegun, National Bureau of Standards, New York, 1964.

[10] M. A. Leontovich, V. A. Fock, Solution of the problem of propagation of electromagnetic waves along the earth's surface by the method of parabolic equation, Journ. of Phys. of the U.S.S.R 10(1) (1946) 13-23.

[11] A. V. Manzhirov, A. D. Polyanin Methods of solving integral equations, Moscow, Factorial, 1999 (in Russian)

[12] I.J. Thompson, A.R. Barnett, COULCC: A continued-fraction algorithm for Coulomb functions of complex order with complex arguments, Comput Phys Commun, 36 (1985) 363-372.

[13] V. A. Fock, Diffraction of radio-waves around the Earth's surface, Journ. of Phys. of the U.S.S.R 9, N 4 (1945) 255.

[14] J. C. Nédélec, Mixed finite elements in $\mathbb{R}^{3}$, Numer. Math., 35, N 3 (1980) 315-341.

[15] P. Monk, Finite element methods for Maxwell's equations, Oxford University Press, 2003. 